\title{
Panencephalopathic type of Creutzfeldt-Jakob disease: primary involvement of the cerebral white matter
}

\author{
TOSHIO MIZUTA NI, *ATSUSHI OKUMURA, $\dagger$ \\ MASAYA ODA, $\ddagger$ AND HIROTSUGU SHIRAKI
}

From the Clinical Neuropathology, Tokyo Metropolitan Institute for Neurosciences, Fuchu,* the Department of Psychiatry, School of Medicine, Osaka City University, $\dagger$ the Department of Neuropathology, Institute of Brain Research, School of Medicine, University of Tokyo, $\ddagger$ and the Shiraki Institute of Neuropathology, Tokyo, Japan§

S U M MARY Eight necropsy cases of a "panencephalopathic" type of Creutzfeldt-Jakob disease (CJD) in the Japanese are reported. The reasons why this type should be discussed separately from other types of CJD are that there is primary involvement of the cerebral white matter as well as the cerebral cortex, and that the white matter lesion of one Japanese human brain with CJD similar to the present group has been successfully transmitted to experimental animals.

Creutzfeldt-Jakob disease (CJD) is a diffuse degeneration of the central nervous system usually occurring in middle age and fatal within two years. Clinical variations are numerous, but the most common picture includes severe mental deterioration, rigidity of muscles and myoclonic jerks. Neuropathological alterations, are widespread and variable, but are confined to the cortical and subcortical grey matter. The white matter is considered never to be a main site of pathology, although demyelination or myelin pallor of the white matter has been described infrequently in some reported cases ${ }^{12}$; such involvement has been interpreted as secondary to neuronal disintegration. ${ }^{3}$

The eight necropsy cases of CJD described here showed severe and extensive degeneration of the cerebral white matter which could not be explained simply as secondary to cortical deterioration. ${ }^{4}$ Such cases with prominent lesions of white matter have not so far been described from western countries. This severe involvement of the white as well as the grey matter explains the marked decrease in brain weight, which was usually below

Address for reprint requests: Dr Mizutani, Department of Clinical Neuropathology, Tokyo Metropolitan Institute for Neurosciences, 2-6 Musashidai, Fuchu-City 183, Japan.

Accepted 30 August 1980
$1000 \mathrm{~g}$, irrespective of the duration of the clinical course ( 5 to 30 months). This finding differs from previous reports that the brain atrophy in CJD is only mild to moderate in degree and increases with the duration of illness. ${ }^{5}$

\section{Clinical findings}

The patients were six males and two females. The age at death was from 47 to 73 years, (average of 58), and the disease occurred particularly in the 5th and 6th decades. The total duration of illness was approximately $5,7,7.5,8.5,9$ and 11 months, and 2 years and 2.5 years. Most ran a subacute clinical course within one year, except cases 7 and 8 which continued for 2 years and 2.5 years, respectively. All cases were sporadic, without significant hereditary factors, and no precipitating cause was evident. There were three stages in the clinical course: the initial or prodromal stage, the intermediate stage of rapid neurological and mental deterioration, and the terminal vegetative state (fig 1 ).

The initial stage was characterised by insidious onset of various physical and psychotic complaints, such as headache, fatigability, loss of appetite, restlessness and mental slowness in most cases, but in cases 2 and 6 the initial disturbance was a sudden onset of diplopia. In case 8 , gradually increasing tremor of the hands was the first complaint. During this stage, numbness ascending from the legs to the arms occurred in case 1 and hand tremor in case 4. This stage lasted for two weeks or less. 
Table Clinical picture of the present group of CJD

\begin{tabular}{|c|c|c|c|c|c|c|c|c|}
\hline & Case 1 & Case 2 & Case 3 & Case 4 & Case 5 & Case 6 & Case 7 & Case 8 \\
\hline Age (yr) & 47 & 55 & 63 & 73 & 62 & 56 & 56 & 49 \\
\hline Sex & $\mathbf{F}$ & $\mathbf{M}$ & F & $\mathbf{M}$ & $\mathbf{M}$ & $\mathbf{M}$ & $\mathbf{M}$ & $\mathbf{M}$ \\
\hline Duration (M) & 5 & 7 & $7 \cdot 5$ & $8 \cdot 5$ & 9 & 11 & 24 & 30 \\
\hline Dementia & + & + & + & + & + & + & + & + \\
\hline Focal signs & & + & & + & + & + & & \\
\hline Perservation & & + & + & + & & & & \\
\hline Hallucinations & & + & & & & + & + & \\
\hline or delusions & & + & & & & & + & \\
\hline Psychomotor excitement & + & + & & & + & + & & \\
\hline Akinetic mutism & + & + & + & + & + & + & + & + \\
\hline Pyramidal signs amyotrophy & + & & & + & + & & & \\
\hline Rigidity & + & + & + & + & + & + & + & + \\
\hline Myoclonic jerks & + & + & + & + & + & + & + & + \\
\hline Tremor & + & & & + & & & & \\
\hline Choreo-athetoid & + & & & & + & + & & \\
\hline Gait disturbance/ataxia & + & + & + & + & + & + & + & + \\
\hline Romberg's sign & + & & & & & + & & \\
\hline Sensory disturbance & + & & & & + & + & & \\
\hline Visual disturbance & & + & & & & + & & \\
\hline $\begin{array}{l}\text { Para-, tetraplegia in flexion/ } \\
\text { extension }\end{array}$ & + & + & + & + & + & + & + & + \\
\hline PSD & + & + & + & + & + & + & + & + \\
\hline Brain weight (Grams) & 1200 & 980 & 990 & 1100 & 1100 & 930 & 1200 & 760 \\
\hline
\end{tabular}

$(+)=$ Present. PSD $=$ Periodic synchronous discharge.

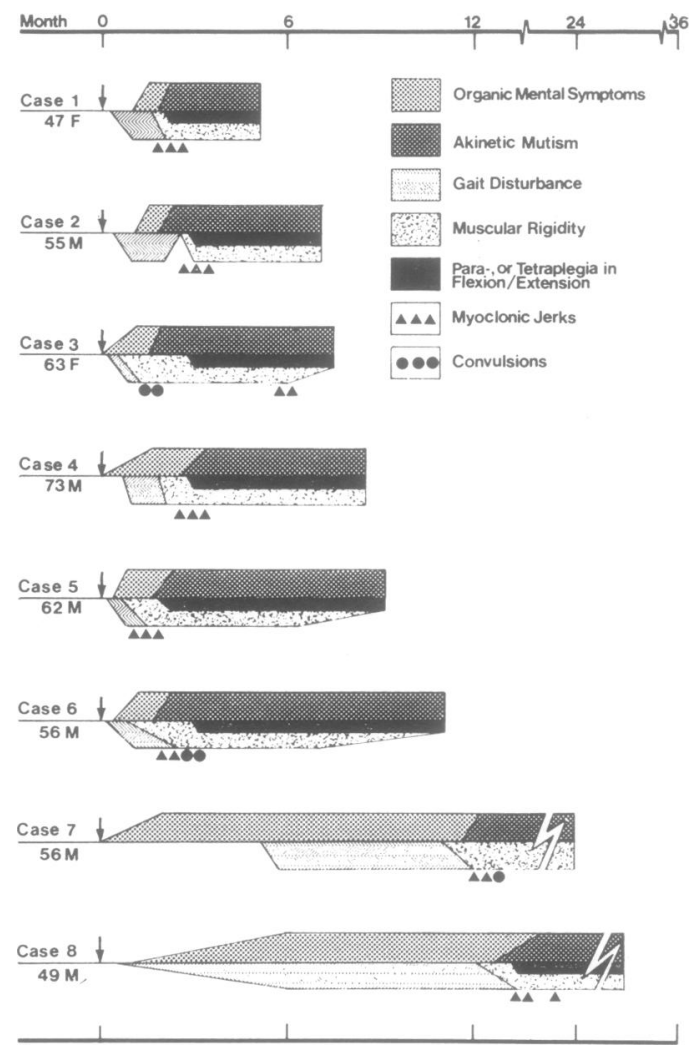

Fig 1 Clinical course of the present group of CJD. Arrow indicates the onset of the disease.
The intermediate stage was of rapid deterioration with neurological and mental disturbances developing one after another and the patients eventually becoming hypokinetic and mute. Although the onset of this stage was not always obvious, rapid development of gait disturbance predominated in cases $1,2,5$ and 6 , while dementia preceded the manifestation of gait disturbance in case 7. Gait disturbance developed sooner or later in all cases, but it was difficult to decide whether it was of pyramidal, extrapyramidal or cerebellar origin. Ataxic gait was observed in cases 1, 2, 4, 5 and 6 and Romberg's sign was evident in cases 1 and 6 . Subsequent rapidly progressive development of muscular rigidity of all extremities soon rendered the patients bedridden. Myoclonic jerks and muscular rigidity developed in all cases; convulsive seizures of grand mal type subsequently occurred in cases 6 and 7. In case 3 , on the other hand, myoclonus was only observed in the terminal stage, while convulsions occurred in the intermediate stage. Periodic synchronous discharges on EEG were recorded before or after the manifestation of myoclonus in all cases.

Tremulous movements of hands were observed in cases 1 and 4. Dysarthria manifested in five cases (cases 1, 2, 4, 5 and 6), but there still remained the question whether this was related to brainstem or cerebellar involvement. Generally speaking, typical symptoms and signs of cerebellar involvement never comprised a main feature in any of the patients, even when severe cerebellar involvement was found postmortem. Pyramidal signs and symptoms, such as hyperactive deep tendon reflexes and pathological reflexes, were observed in cases 1,4 and 5 , while neurogenic muscular atrophy was never encountered. Choreoathetoid movements were found in cases 1,5 and 6 . The main psychic disturbance was progressive de- 
mentia. Various cerebral focal signs, such as aphasia, apraxia and agnosia, also were observed, although they were sooner or later superseded by steady rapid progression of global dementia. In addition, psychomotor excitement, delusion and visual hallucination occurred transiently during the course of illness.

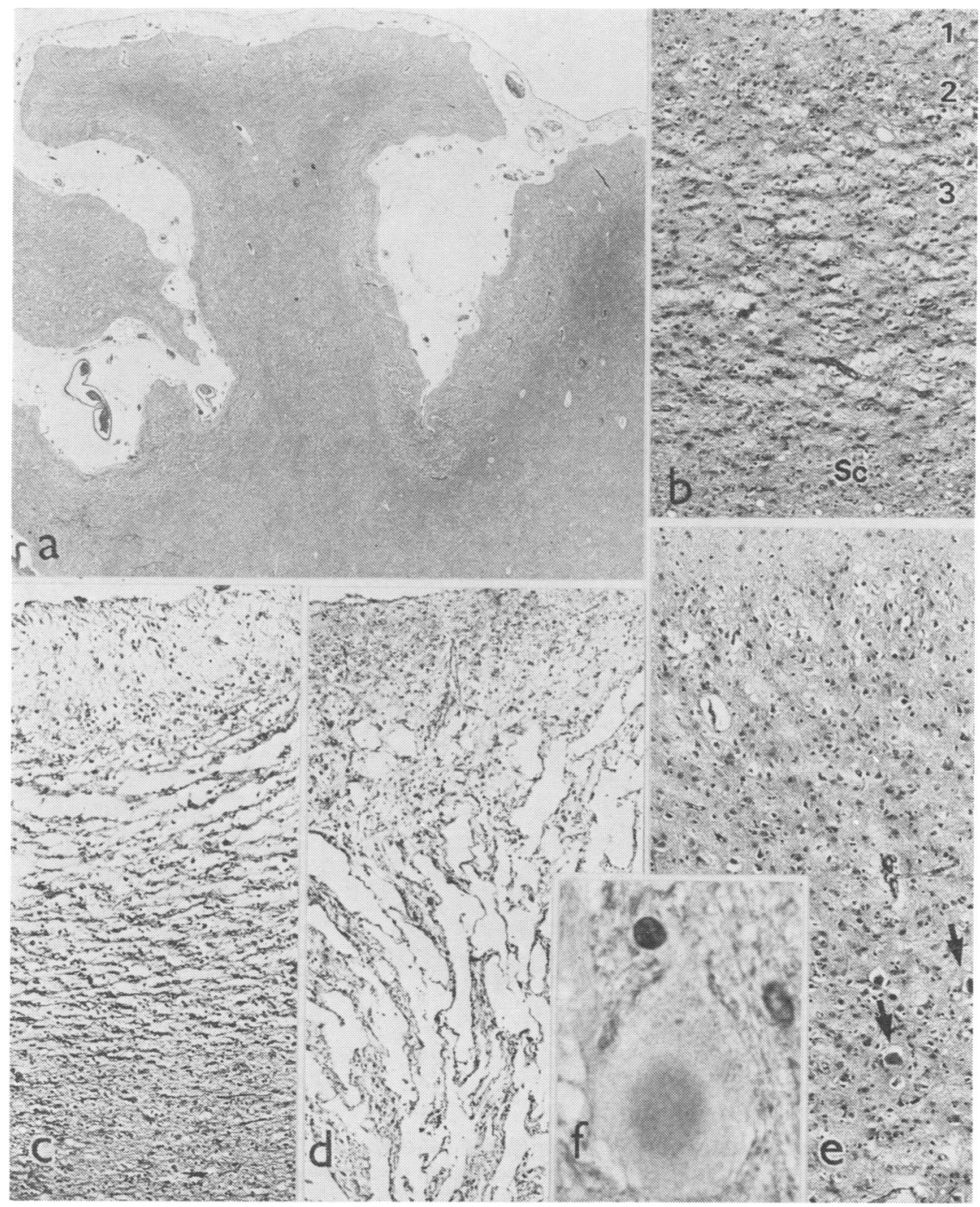

Fig 2 a: Cerebral cortex of the frontal pole. Severe cortical atrophy, particularly in the depths and walls of the conspicuously dilated sulci (case 6). b: Frontal cortex in the wall of the sulcus. Severe neuronal loss, conspicuous proliferation of astrocytes and sieve-like tissue loosening in almost all lavers, except the molecular layer. 1: The molecular layer; 2: The second layer; 3: The third layer; Sc: Subcortical white matter (case 6). c: Frontal cortex in the depth of the sulcus. Pronounced tissue destruction of laminar distribution in almost all layers, except the molecular layer (case 8). d: Temporal cortex. Microcystic coalescent cavitations in almost all layers, except for the molecular layer, continuously involving the subcortical white matter (case 8). $e:$ Frontal cortex at the crown of the gyrus. Comparatively well preserved neurons in contrast to proliferated astrocytes. Inflated neurons in the deeper layers (arrows) (case 6). f: Deeper layer of insular cortex. Marked inflated neuron with an eosinophilic intracytoplasmic inclusion (case 3). [a, b, c, d,e and $f: H \& E ; a: \times 4 \cdot 8$ b: $\times 64 ; c: \times 64 ; d: \times 32 ; e: \times 64 ; f: \times 640]$. 


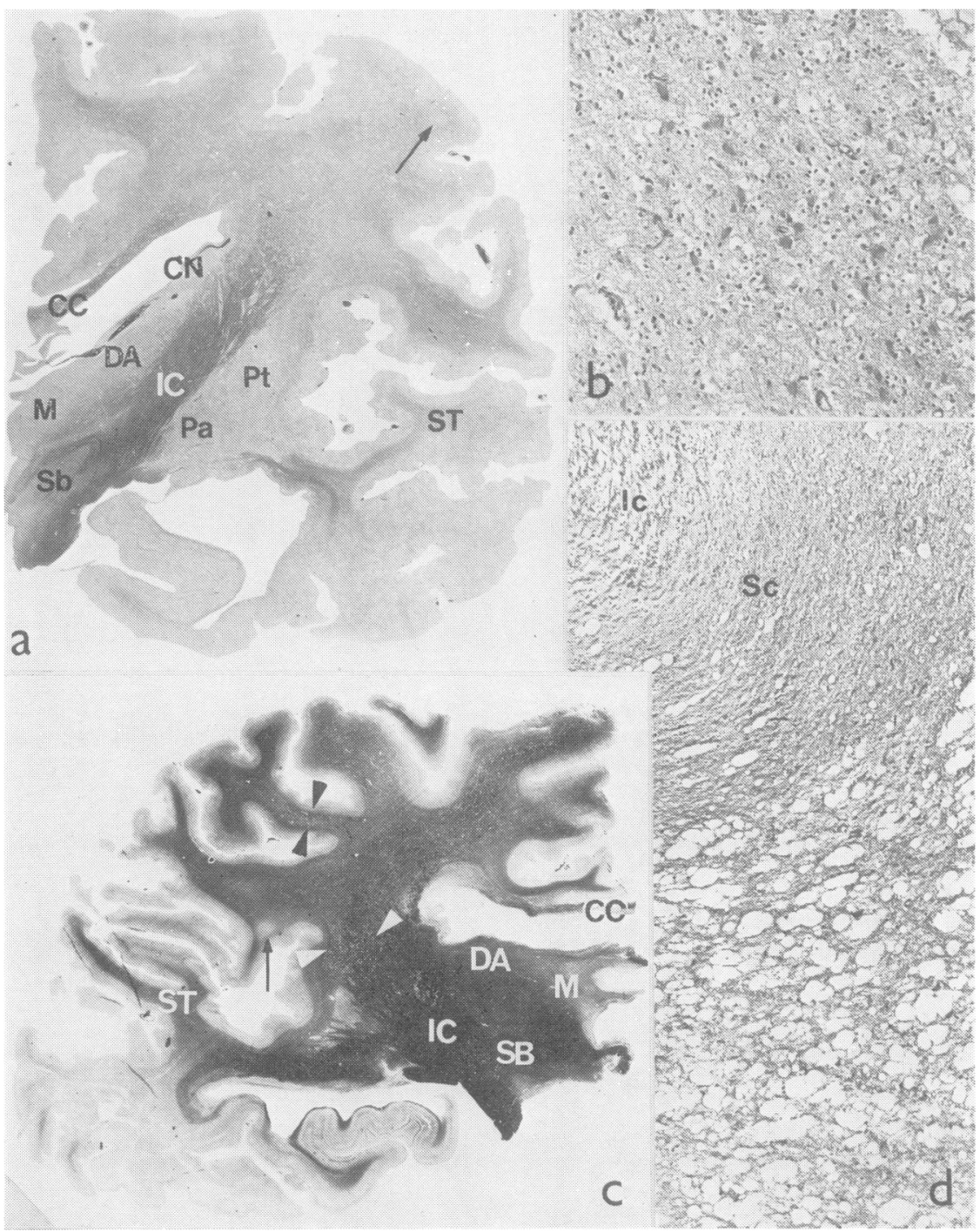

Fig 3 a: Frontal-cut hemisphere through the subthalamus $(S b)$. Diffuse pallor of the centrum semiovale in contrast to the preservation of the internal capsule (IC). Thinning of the corpus callosum (CC). Demyelination of the temporal white matter with relatively better preservation of the superior temporal white matter $(S T)$. Disintegrated myelinoarchitecture in the caudate nucleus $(C N)$, putamen $(P t)$, Pallidum (Pa), and fomatio medialis $(M)$ and nucleus dorsalis anterior $(D A)$ of the thalamus. Arrow indicates circumscribed spongy focus. Well preserved hippocampal formation (case 6). b: Centrum semiovale in a. Marked loosening or spongy state, pronounced hypertrophic and gemistocytic astrocytes, and a large number of fat granule cells (case 6). c: Frontal-cut hemisphere through the subthalamus $(S B)$. In addition to similar findings to a, coarse spongy cavitations in the subcortical white matter (black arrow-heads) and in the dorsal portion of the internal capsule (white arrow-heads). CC: Corpus callosum; ST: Superior temporal white matter; IC: Internal capsule; M: Formatio medialis thalami; DA: Nucleus dorsalis anterior thalami (case 8). $d:$ Coarse spongy cavitations in the dorsal portion of the internal capsule in $c$. Multiple, solitary and confluent spongy cavities and proliferated gemistocytic astrocytes between. Spared subcortical white matter $(S c)$ and marked devastation of the insular cortex (Ic) (case 8). [a: $K-B, \times 1 \cdot 3 ; b: H-E, \times 82 ; c: K-B, \times 1 \cdot 2$; $d: H-E, \times 32]$. 


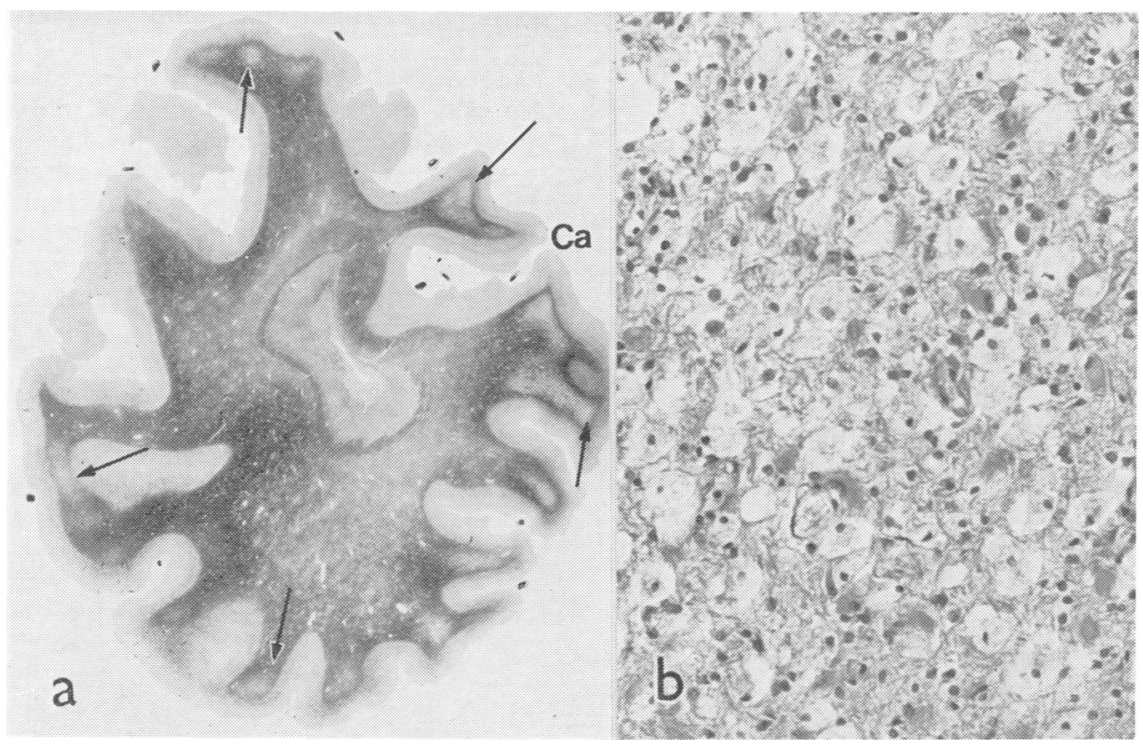

Fig 4 a: Occipital lobe. Multiple, circumscribed spongy foci scattered in the subcortical white matter at the crowns of the gyri (arrows). Coalescent or ill-defined patchy pallor of the deep white matter as well. Ca: Calcarine sulcus (case 5). $b$ : Numerous spongy cavities filled with gitter cells and proliferated gemistocytic astrocytes between (case 7$)$. [a: $K-B, \times 1 \cdot 8 ; b$ : $\times 224]$.

The transition from the intermediate to the terminal stage was not always obvious, since the patients became gradually akinetic and mute. Nevertheless the duration of the intermediate stage was two to three months after the onset of the illness to the appearance of akinetic mutism in six cases, and one year in cases 7 and 8 (fig 1). The duration of the terminal stage varied, from 3 months to 1.5 years. The extremities gradually developed paraplegia or tetraplegia in flexion or extension. Muscular rigidity more or less continued to the time of death, but in cases 3,5 and 6 it gradually changed to flaccidity. After akinetic mutism and generalised posture flexion appeared, the course became rather stationary. Nasogastric tube feeding inevitably was necessary. Various intercurrent infections developed in association with difficulty of swallowing, incontinence of urine and decubitus, and death occurred, as a rule, with an intercurrent illness in a cachectic state. Although cases 7 and 8 experienced a long illness, particularly in the intermediate stage, their clinical picture was essentially identical to that of the other cases.

\section{Laboratory findings}

Periodic synchronous discharge on EEG was recorded in all cases, during the stage of myoclonic jerks. No significant findings were found in the blood and cerebrospinal fluid. In cases 2 and 4, examination of the urine and hair for heavy metals, such as lead and mercury, showed no pathological values.

\section{Neuropathological findings}

The brain weight ranged from $1200 \mathrm{~g}$ to $760 \mathrm{~g}$. Both cerebrum and cerebellum showed moderate to conspicuous atrophy, particularly in the cases with the brain weight below $1000 \mathrm{~g}$. The ventricles were dilated symmetrically and bilaterally to various degrees. The cerebral cortex, except for the hippocampal formation and amygdala, was extremely thin, and showed laminar tissue disruptions. Cerebral atrophy was more marked in the frontal, temporal and occipital lobes and in the lateral convexity than in the central region and the medial surface. The cerebral white matter was soft and swollen but no obvious cystic formation was seen. The striatum and thalamus were also atrophic. The cerebellum showed generalised and severe cortical atrophy. There were no remarkable findings in other viscera, except for occasional bronchopneumonia and central fatty infiltration of the liver.

\section{Microscopic findings}

The cerebral cortex showed widespread severe cortical lesions in both hemispheres of all cases, summarised as follows:

(1) The cortical changes were, as a rule, extensive and severe involving almost all cerebral gyri in variable degrees (figs 2a, 3a, 3c and 4a). They were particularly pronounced in the depths and walls of the sulci, affecting all layers except for the molecular (figs 2a and 9) and consisted of severe loss of nerve cells, conspicuous proliferation of hypertrophic or 


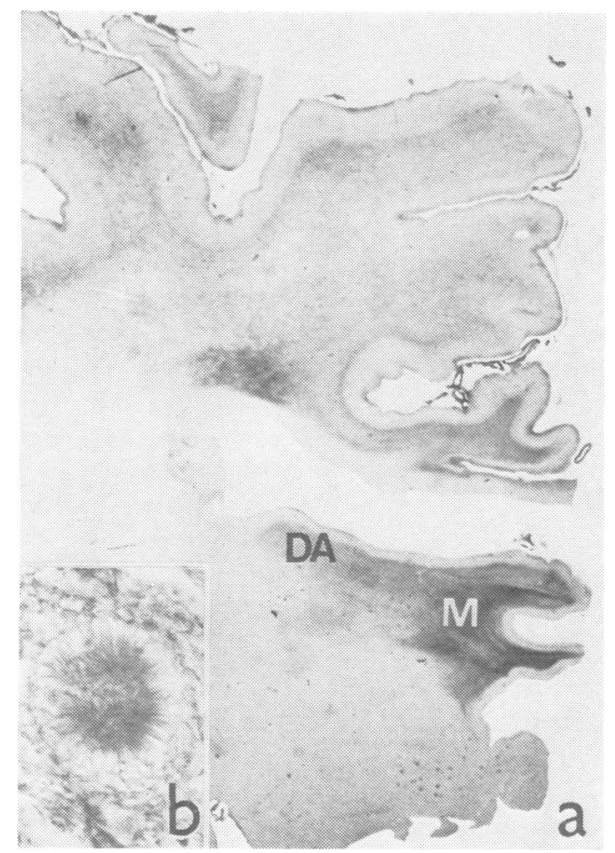

Fig 5 a: Conspicuous fibrillary gliosis in the formatio medialis $(M)$ and less intense in the nucleus dorsalis anterior $(D A)$ of the thalamus. Mild fibrillary gliosis in the digital white matter as well (case 8). b: One typical Kuru plaque in the formatio medialis of the thalamus (case 7). [a: Holzer, $\times 1 \cdot 6 ; b: H \& E, \times 400]$.

gemistocytic astrocytes, and sieve-like tissue-loosening which tended to coalesce in laminar distribution (figs $2 b$ and $2 c)$. In the most severely affected regions, microcystic cavitations occurred in almost all layers, except for the molecular layer, and continuously involved the subcortical white matter in a similar manner (fig 2d). Similar but less severe changes were evident at the gyral crowns usually in a laminar fashion, particularly in the third layer. The hippocampal formation and amygdala were, as a rule, spared.

(2) In these cortical lesions, abundant small sized fat droplets were phagocytosed mainly by activated microglial or gitter cells, but there was little tendency for perivascular accumulation. In spite of conspicuous astrocytosis, no compact proliferation of slendercalibre gliofibres occurred, irrespective of differences of the clinical course from 5 to 11 months.

(3) In the mildly affected cortical areas, the nerve cells were comparatively well-preserved in contrast to pronounced proliferation of glial nuclei (fig 2e). The neuropil of the cortex was finely loosened or disrupted, but no clustered grape-like cavitations were found anywhere. Nerve cells, particularly in the deeper layers, showed a swollen and pale cytoplasm with eccentric nucleus, some of which contained an eosinophilic, ill defined intracytoplasmic inclusion (fig 2f).

The cerebral white matter in myelin sheath preparations (figs $3 \mathrm{a}$ and $3 \mathrm{c}$ ), showed diffuse and widespread pallor which prevailed not only in the centrum semiovale, but also in the frontal, temporal and occipital white matters. The internal capsule was spared in all cases. Axons were obviously decreased in number. The white matter became conspicuously loosened or spongy (fig 3b). Abundant fat droplets were observed not only in the phagocytic cells exclusively of a fixed type, but also in the hypertrophic astrocytes. Proliferation of hypertrophic and gemistocytic astrocytes was less pronounced than that in the cortex, but fibrillary gliosis was never encountered. In most cases, the severity of both cortical and white matter degeneration was almost equal (fig 9). It must be emphasised also that, apart from this diffuse lesion, sharply-defined spongy foci were found preferentially in the subcortical white matter of the gyral crowns, where U-fibres or the deeper cortical layers or both were usually spared, (figs 3a, 3c and 4a). A large number of fat droplets were phagocytosed by activated microglial and/or gitter cells (fig 4b), while dark shrunken, presumably astrocytic, nuclei were rarely in these foci. A great majority of these foci were represented only by the empty spaces. A conspicuous proliferation of astrocytes, many of which were gemistocytic, was found in the foci from which both myelin sheaths and axons had disappeared almost completely. In case 8 , such foci and also coarse spongy cavities occurred in the subcortical white matter and in the dorsal portion of the internal capsule (figs 3c and 3d). Similar spongy foci were found in the lateral columns of the spinal cord in case 6 (fig 7) which will be described later.

Basal ganglia and thalamus (1) The caudate nucleus, putamen and claustrum showed identical alterations to the cortical deterioration mentioned above (fig 3a). The globus pallidus, on the other hand, usually showed no outstanding alterations, although a diffuse pallor and proliferation of glial nuclei occurred (fig 3a).

(2) In all cases, the thalamic subnuclei were damaged bilaterally. Although the severity of the lesions in each subnucleus varied from case to case, both the formatio medialis and dorsal portions of the nucleus dorsalis anterior and posterior were the commonest sites (fig 9) and particularly in cases 7 and 8. Although identical to the cortical alterations, neuronal loss was less severe (figs $3 \mathrm{a}$ and $3 \mathrm{c}$ ) and $\mathrm{a}$ very few Kuru plaques were found (fig 5 b). The pulvinar thalami disclosed similar but milder degeneration. Other subnuclei were less severely involved or not damaged, although astrocytosis was diffuse.

The cerebellar changes of similar quality and quantity were consistent in all eight cases.

(1) The granular layer was most susceptible and the granule cells were severely disintegrated with pronounced spongy tissue loosening and proliferation of 


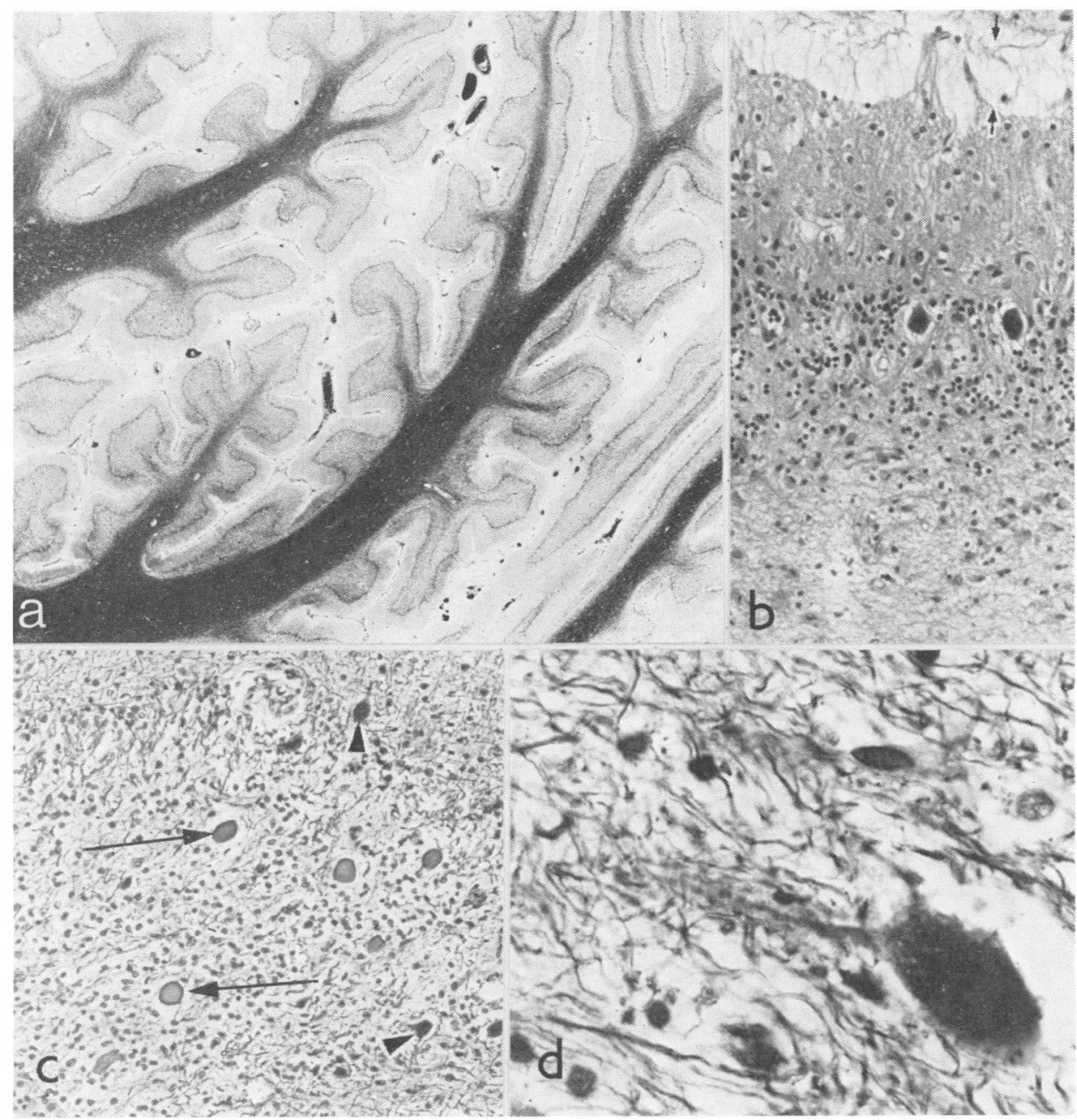

Fig 6 Cerebellum. a: Cortical degeneration of granule cell type in all folia (case 5). b: Subpial rarefaction of the molecular layer (arrows), conspicuous loss of the granule cells with proliferated hypertrophic astrocytes, and well-preserved Purkinje cells (case 6). c: Many torpedo formations (arrows) in the granular layer. Arrow-heads indicate Purkinje cells (case 5). d: Dentate nucleus. "Grumose alteration" mainly of peridendritic and less of pericytoplasmic areas of neuron consisting of deeply argentophilic solid bodies and ring-shaped ones, and faintly argentophilic fibrillo-granular structures (case 6). [a: $K-B, \times 6 \cdot 4 ; b: H \& E, \times 160 ; c:$ Bodian, $\times 160 ; d$ : Bodian, $\times 640]$.

hypertrophic astrocytes (fig 6b). These changes were exceedingly widespread and bilaterally symmetrical in almost all folia of paleocerebellum and neocerebellum (figs $6 \mathrm{a}$ and $8 \mathrm{a}$ ). The crowns of the folia were more affected than the depths. Both isomorphic and anisomorphic gliosis with slender-calibre gliofibres occurred rarely in the molecular and granular layers. The astrocytic proliferation and hypertrophy occurred also in the white matter, predominantly in the subcortical areas, which were sometimes conspicuously disrupted as well. The Purkinje cells, on the other hand, were rather well preserved and the proliferation of the Bergmann's glial nuclei was equivocal (fig $6 \mathrm{~b}$ ). The axonal torpedos of Purkinje cells were found frequently (fig 6c). The molecular layer disclosed a subpial and laminar rarefaction, particularly severe in cases 6,7 and 8 , but there was no microcavitation (fig 6b). There were many phagocytic cells laden with neutral fat granules in all cortical layers, predominantly in the molecular layer. The grade of their mobil- 


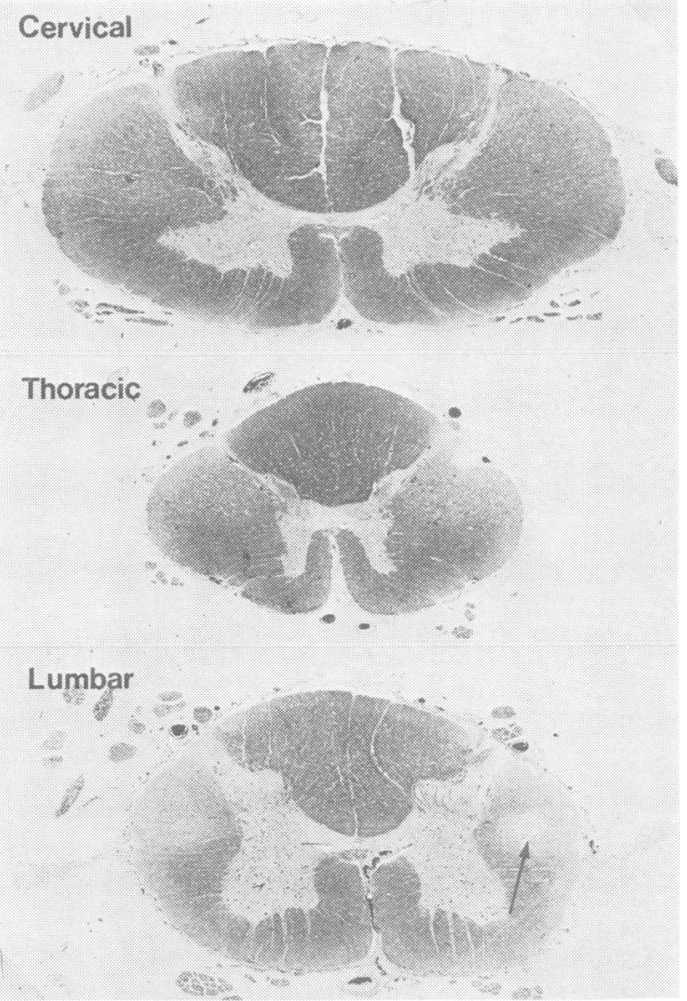

isation varied from case to case, while no perivascular accumulation occurred as seen in the cerebral lesions.

(2) In the dentate nucleus, the nerve cells were well preserved and occasionally developed perineuronal "grumose alteration" (fig 6d) in cases 1, 2, 4 and 6, the details and significance of which have been discussed elsewhere..$^{-8}$

Brainstem and spinal cord (1) The brainstem nuclei showed no remarkable changes except in case 7 which will be described later. (2) Degeneration of the corticospinal tracts was found in cases 1, 6 and 7 and was obviously more pronounced at the lumbar level than at the cervical level (fig 7). In the lateral columns of the lumbar cord in case 6, there occurred sharply demarcated foci of aggregated empty cavities which disclosed a large number of fat droplets phagocytosed by microglial cells and astrocytic proliferation. In these foci, not only axons of small calibre but also of large calibre almost completely disappeared, while in other foci of lateral columns, axons of small calibre still remained.

Fig 7 Bilateral corticospinal tract degeneration of distal character. Arrow indicates circumscribed spongy focus in the lateral column of the lumbar cord (case 6). [K-B, $\times 6 \cdot 6]$.

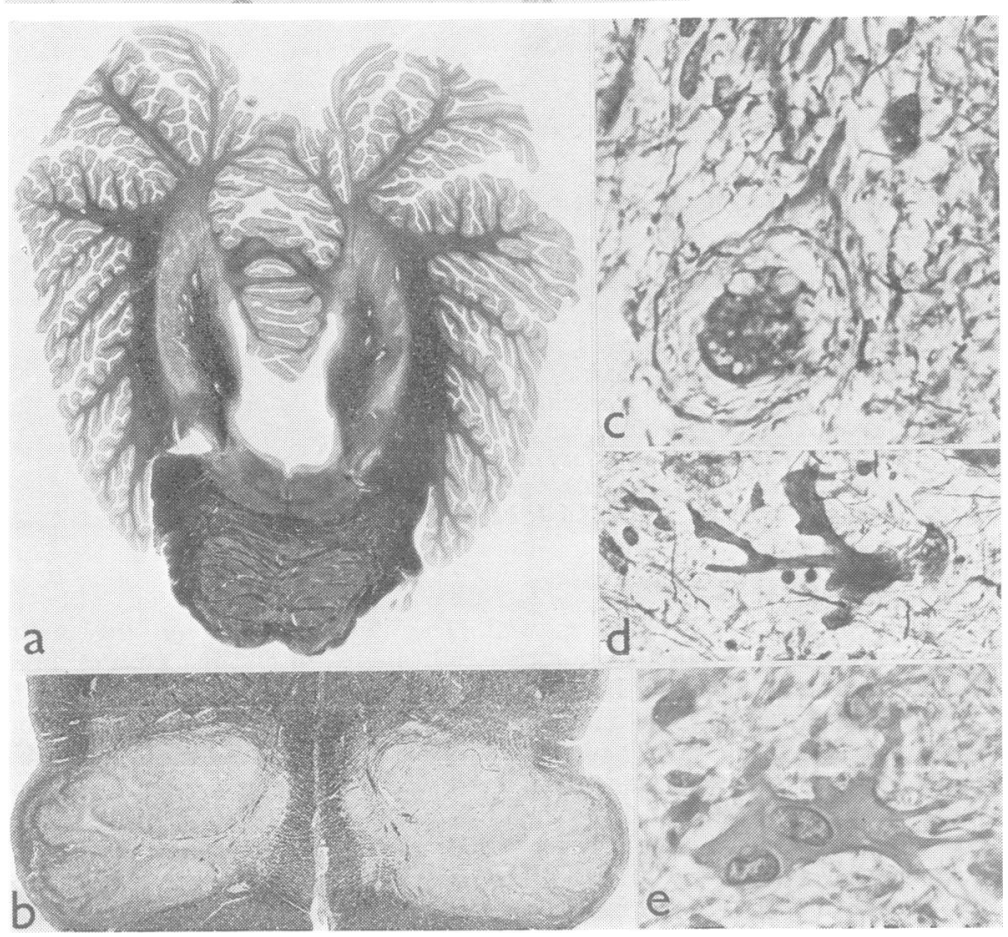

Fig 8 Case 7. a: Bilateral and symmetric cerebello-cortical degeneration. Moderate to severe demyelination of the white matter mainly around the dentate and less pronounced around other deep nuclei. $b$ : Hypertrophy of the inferior and medial paraolivary nuclei, and marked demyelination particularly in the hilus and less in the fleece. c: Inferior olive in $b$. Tangled argentophilic fibres around a nerve cell with multiple intracytoplasmic vacuoles. $d$ : Inferior olive in $b$. Conspicuously thickened dendrites of the nerve cell. $e$ : Inferior olive in b. Giant astrocyte with two nuclei. $[a: K-B, \times 1 \cdot 1 ; K-B, \times 3 \cdot 8$; c: Bodian, $\times 1152 ; d$ : Bodian, $\times 440 ; e: H \& E, \times 640]$. 


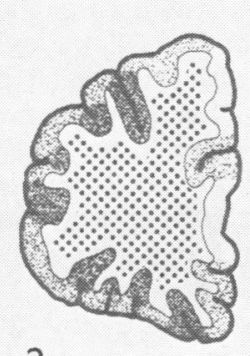

a
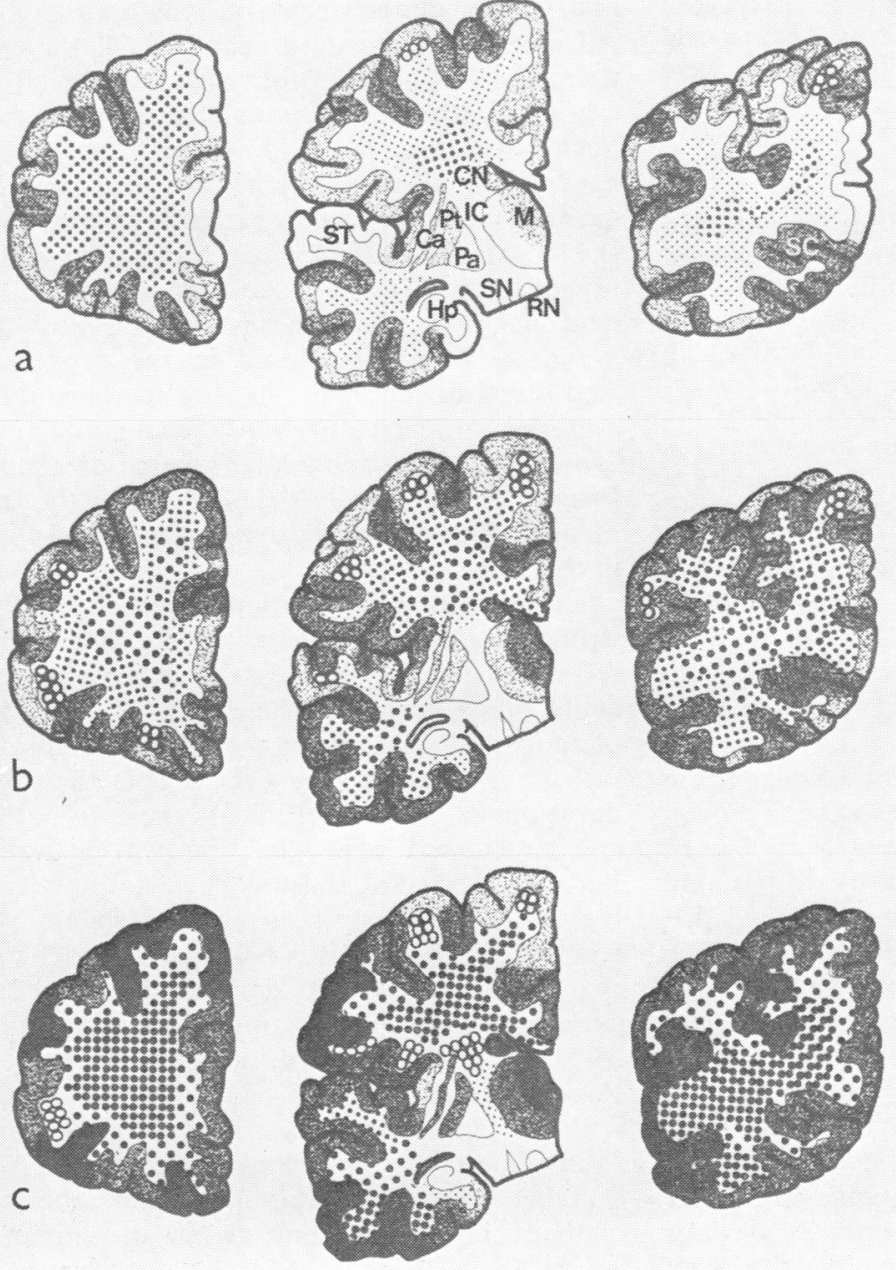
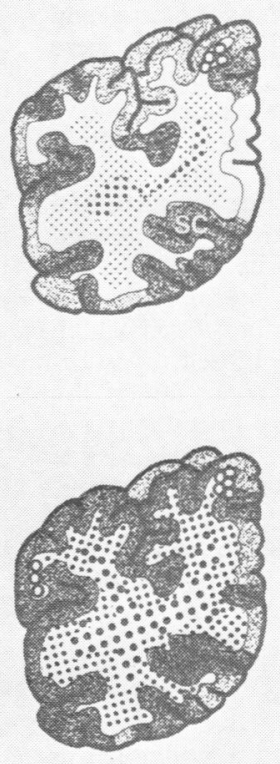

Fig 9 Schematic distribution of the cerebral lesions in 3 cases of the present group. a: Case 1. 47-year-old female. Five months' duration. Brain weight, $1200 \mathrm{~g}$. b: Case 6. 56-year-old male. 11 months' duration. Brain weight, $930 \mathrm{~g}$. c: Case 8. 49-year-old male. 30 months' duration. Brain weight, 760 g. M: Formatio medialis of the thalamus; Pt:Putamen; Pa: Pallidum; Ca: Claustrum; SN: Substantia nigra; $R N$ : Red nucleus; Hp: Hippocampus; IC: Internal capsule; ST: Superior temporal gyrus; SC: Striate cortex.
Cortical lesions

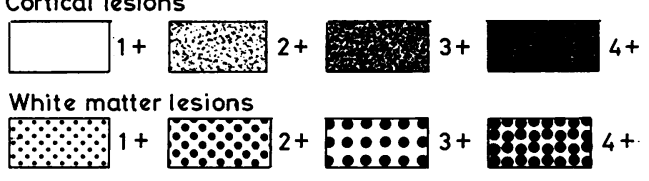

Subcortical spongy foci

\section{8}

\section{Additional findings}

(1) The formatio medialis in the thalamus of cases 7 and 8 disclosed complete loss of neurons and intense fibrillary gliosis (fig 5a).

(2) The lateral geniculate bodies in cases 6 and 7 were severely damaged bilaterally. The nerve cells were disintegrated almost completely, the astrocytes proliferated intensely, and the fat granule cell mobilisation was found out. Furthermore, in case 6 , both optic tracts were markedly degenerated, and in case 7 , the optic radiations of the occipital white matter were severely disrupted and a large number of fat granule cells were mobilised as compared with the less severely affected adjacent white matter.

(3) A marked olivary hypertrophy occurred symmetrically and bilaterally in case 7 (fig 8b). In myelin sheath preparations, prominent demyelination involved intensely the enlarged inferior olivary nucleus and less intensely the medial paraolivary nucleus as well as the whole areas of the adjacent white matter. Many nerve cells of these nuclei disappeared, while the cytoplasm of the remaining neurons became markedly inflated. Some of them developed intracytoplasmic single or multiple vacuoles (fig 8c). Thickened dendritic processes of the remaining nerve rells and 
tangles of argyrophilic fibres around the nerve cell were observed (figs 8c and 8d). Many bizarre and giant astrocytes with single or multiple nuclei were seen (fig 8e). The central tegmental tracts at the pontine level disclosed no particular changes. Nerve cells of the dentate nucleus and less obviously those of other deep cerebellar nuclei were bilaterally disintegrated to a slight to moderate degree accompanied by marked astrocytosis. The comparatively well defined demyelination developed in the white matter symmetrically and bilaterally along the lateral border of the dentate and emboliform nuclei (fig 8a), in which large number of gitter cells were mobilised. This became more pronounced in the ventral portion of the dentate nucleus and was continuous into the restiform body. The pallor of the myelin was also visible in the hilus closely adjacent to the dentate nucleus. The brachium conjunctivum was not involved.

(4) In case 7 , only one typical Kuru plaque was found in the formatio medialis of the thalamus, in only one section (fig $5 b$ ).

\section{Discussion}

The term CJD is used nowadays in a wider sense than in the days of Creutzfeldt ${ }^{9}$ and Jakob's ${ }^{10}$ original descriptions. Particularly since transmission of subacute spongiform encephalopathy (SSE) and Kuru to experimental animals has succeeded, ${ }^{11}$ SSE has become almost synonymous with CJD. From the clinicopathological point of view, however, so-called classical CJD, based on the original cases reported by Creutzfeldt ${ }^{9}$ and Jakob, ${ }^{10}$ could not be considered to be entirely identical with SSE, ${ }^{12} 13$ although it is apparent that both conditions share several common clinical and pathological features. ${ }^{514}$ It therefore seems to us that CJD now includes a wide spectrum with various clinical and pathological features, and the term SSE is representative of only one subtype of CJD.

The disease group presented here came on subacutely in the fifth and the sixth decades, and the total duration of illness ranged within one year in most cases with a rapid downhill course. In two patients (cases 7 and 8 ) the clinical course was exceptionally protracted for two years and two and a half years, respectively. The clinical picture included various neurological and mental disturbances, the following were found consistently in all eight cases: severe mental deterioration leading to akinetic mutism, paraplegia or tetraplegia in flexion and/or extension, muscular rigidity, myoclonic jerks, gait disturbance and periodic synchronous discharge in the EEG. The cerebellar signs and symptoms, on the other hand, were never a main feature, particularly from intermediate to terminal stage, in contrast to Brownell and Oppenheimer's cases in which cerebellar impairments lasted until death. ${ }^{15}$ Pathologically, there was not only severe cerebrocerebellar degeneration with conspicuous astrocytosis without fibrillary gliosis, but also severe white matter degeneration. Cerebello-cortical degeneration of granule cell type occurred without exception. In the less severely affected cerebral cortex and in the hippocampus, astrocytosis predominated, while neuronal loss was less pronounced and inflated nerve cells were scattered in the deeper layers. The cortical changes in the cerebellum were almost identical to or more severe than those in Brownell and Oppenheimer's cases of the ataxic form of CJD, ${ }^{15}$ while the severity of the involvement of the cerebral cortex far exceeded that seen in their cases.

The cortical degeneration in the present group strongly suggests that a rapid development of severe destructive process occurred even at the acute stage, because there existed almost complete loss of neurons with marked mobilisation of fat granule cells, irrespective of the different durations of the total clinical course in both acute and protracted cases. It, however, is noticeable that a majority of these cortical lesions were not typical of the clustered grape-like spongy state in SSE. In this regard, it is possible that such atypical changes in SSE are derived from collapsing phenomena of pre-existing status spongiosus. ${ }^{16}$ Masters and Richardson, on the other hand, mentioned that these lesions semed to be comparable with non-specific status spongiosus which may be found in various pathological conditions. ${ }^{17}$ It still remains an open question whether the cortical changes in the present group are different in origin from those in SSE or not. Nevertheless, minor neuronal changes in some cortical areas, in spite of diffusely proliferated astrocytes and spongy tissue loosening, do not always allow a clear-cut distinction to be made between the present group and typical SSE.

A great discrepancy between the present group and SSE, however, does exist in that the widespread severe degeneration of the cerebral white matter in the present group has never been encountered in SSE or in other necropsy reports of CJD in western countries. It is true that, although some overlapping process of the secondary degeneration attributed to the severe cortical damage did exist in the white matter, much of it was regarded as primary for the following reasons: (1) The circumscribed spongy foci, occurring not only in the subcortical white matter with preservation of U-fibres but also in the internal capsule (case 8) 
and the lateral columns of the lumbar cord (case 6), could not be explained as secondary degeneration. (2) The pyramidal tract and the internal capsule themselves were never involved, while the centrum semiovale was severely involved (cases 2 , 3,4 , and 5). (3) The pyramidal tract degeneration in cases 1, 6 and 7 was clearly distal in character and could not simply be attributed to secondary degeneration alone. (4) Review of the literature shows cases in which, in spite of severe cortical devastation, the medullary degeneration was mild or even absent. ${ }^{13}$ 18-20

These findings explain why the present disease group has been named the panencephalopathic type of CJD. There is further support in the results recently obtained by Tateishi and his collaborators who were successful in transmission from one human necropsy case of CJD with dominant involvement of the cerebral white matter to small animals; the pathological alterations in the animals were found not only in the grey matter but also or predominantly in the white matter of the cerebrum, the cerebellum and the brainstem. ${ }^{21}$ That patient, whose clinical picture was that of CJD, was a 56-year-old male with four years' duration whose brain weighed only $940 \mathrm{~g}$. The cerebral white matter was more atrophied than the cortex and showed extensive softening and cystic cavitations. Cerebello-cortical degeneration of granule cell type and many Kuru plaques also occurred. Furthermore, marked neuronal loss in the inferior olivary, pontine, and thalamic nuclei, and severe degeneration of the spinocerebellar and the corticospinal tracts of spinal cord accompanied by intense fibrillary gliosis were symmetrical and bilateral. The clinicopathological details of this patient have been reported by Ohta et al. ${ }^{22}$ In other Japanese cases of CJD accompanied by multiple Kuru plaques, findings similar to those in this example also were reported recently. ${ }^{23-25}$ In such cases, the white matter lesions were more severe than those in the group of CJD described in our report, and their severity exceeded clearly that of the cortical lesions. Subcortical spongy foci were not obvious or were even absent. From the clinicopathological point of view, there exist several similarities and differences between these cases with multiple Kuru plaques mentioned above and the present group which we call the panencephalopathic type of CJD. (1) In the former cases, the clinical course, as a rule, was conspicuously slow, the duration of illness was much longer (ranging from four to 11 years), and the brain weight was markedly decreased (from $1180 \mathrm{~g}$ to $890 \mathrm{~g}$ ). The brain atrophy in our cases also was conspicuous, but the clinical course was very rapidly progressive and the duration of illness was less than one year in most cases, so the degree of the brain atrophy was not proportional to the length of illness. (2) Definite cerebellar symptomatology in the former cases was a main feature in the early stage of the illness and predominated even in the later stage; corresponding cerebello-cortical degeneration was combined with degeneration of spinocerebellar tracts in all cases. Furthermore, in two of the former cases disintegration of the dentate, pontine, inferior olivary and thalamic nuclei also was found. ${ }^{22-23}$ In our cases, on the other hand, a pronounced clinicopathological dissociation existed: the clear-cut cerebello-cortical degeneration in pathological preparations contrasted with the equivocal cerebellar symptoms and signs, or even their absence in the clinical conditions. (3) Rigidity of muscles in our cases was observed from the initial to the terminal stage and the striatum was bilaterally and severely involved, while in the former cases rigidity was less severe or even absent during the total clinical course and the striatum was only mildly damaged or even spared. (4) In both groups the onset of obvious dementia, resulting in akinetic mutism in the terminal stage, coincided with the disappearance of cerebellar signs and symptoms. However, mental deterioration in our cases started in the initial stage and ran a progressive course; in the former patients, except for one case, ${ }^{22}$ organic mental symptoms were not remarkable in the initial stage and dementia became manifest at the later stage. The nature of damage in both the cerebral cortex and the white matter in the former cases suggested that the pathological process developed very slowly and was far less severe in the initial stage than in our cases. (5) The cerebral cortex and the white matter together with the striatum were bilaterally and severely involved at an early stage in our patients, and these changes may have suppressed the clinical manifestations of cerebellar involvement, which were never a dominant feature. In the former cases, it is suggested that the cerebellocortical and striatal involvement were far less severe at the initial stage and did not suppress the clinical manifestation of cerebellar involvement.

The panencephalopathic type of CJD has never been shown in western literature to include CJD with Kuru plaques. ${ }^{26-30}$ The only case worth referring to here was the example reported by Kirschbaum, who considered this as a transition between CJD and Pick's disease. ${ }^{5}$ The clinical 
picture included progressive mental disturbances resulting in akinetic mutism, rigidity of muscles, myoclonic jerks without periodic synchronous discharge in the EEG, and tetraplegia in flexion. The brain weighed $1250 \mathrm{~g}$. The cerebrum showed generalised and conspicuous atrophy and the cortex was strikingly reduced in width, particularly in the depths of walls of the sulci. In addition, there was a generalised atrophy of the centrum semiovale. The cerebral cortex showed severe neuronal loss, marked astrocytosis and mobilisation of fat granule cells. Some of the remaining neurons showed swollen cytoplasm with weakly argentophilic intracytoplasmic inclusion which conformed to Pick's cells. There were necrotic foci of the subcortical white matter which partly involved U-fibres. The deeper white matter showed diffuse reduction of myelin sheaths, spongy state and isomorphic gliosis. The caudate nucleus and the thalamus showed alterations similar to the cortical changes. Disintegration of the granule cells in the cerebellar cortex also was found out. There was neuronal loss in the brainstem and anterior horn cells combined with corticospinal tract degeneration. The clinical picture and course could well be considered identical with those of CJD. Inflated neurons with simulated intracytoplasmic inclusion were found not only in the our panencephalopathic type of CJD, but also in other SSE cases, but we think that they clearly were different from typical Pick's cells. The diffuse degeneration of the centrum semiovale is uncommon in Pick's disease and localised necrotic foci have never been reported. The case reported by Kirschbaum may have been an example of the panencephalopathic type of CJD.

A few other findings in our group of cases of CJD are worth mentioning briefly. (1) There was a close combination of degeneration of anatomicallyinterrelated systems, such as olivary hypertrophy combined with degeneration of restifom body, dentate and deep cerebellar nuclei, and cerebellar cortex (case 7); and degeneration of lateral geniculate body combined with degeneration of the optic tract (case 6) and optic radiation (case 7). (2) In cases 7 and 8, there was a discrepancy for the formatio medialis of the thalamus showed a marked fibrillary gliosis, while the other grey matter disclosed no outstanding gliosis, in spite of conspicuous astrocytosis. (3) Only one but clear-cut Kuru plaque was found in the thalamus in case 7 , but not in the cerebellar cortex.

Each of these findings is unusual in CJD and it is uncertain whether these changes were coincidently combined with CJD or not. However, all the findings listed above were found in one of the three autopsy cases reminiscent of Kuru reported by Krücke et $a l,{ }^{28}$ although the white matter lesions were never found.

Several subtypes of CJD have been separated on clinical neuropathological grounds by many authors, ${ }^{514}$ 31-34 $^{-3}$ but the question of its classification and pathogenesis still seem to be complicated and uncertain. The present group of CJD shares many features with subtypes of CJD already described, but could be distinguished because it was clearly panencephalic, while other subtypes of CJD affect exclusively the grey matter.

We wish to thank Dr Akai of the Yamanashi Prefectual Hospital (case 1), Dr Akima of Toho University (case 5), Dr Murobushi of the Kikuchi National Hospital (case 3), Professor Oikawa and Dr Tadokoro of St Marianne Medical College (cases 2 and 4), Dr Okada of Ohsaka University (case 8) and Dr Okino of Tokyo Women's Medical College (case 6) for permission to study clinical data and necropsy material. We also wish to thank to Mr Kobayashi and Mr Shoji of the Photographic Center of Tokyo Metropolitan Institute for Neurosciences for technical assistance with the photographs. The work was assisted partly by a grant from the Psychiatric Institute of Jikeikai Hospital.

\section{References}

1 Christensen E, Brun A. Subacute spongiform encephalopathy; Spongiform cerebral atrophy. Neurology 1963; 13:455-63.

2 Kinoshita J, Mizuno R, Yagishita S, Iida M, Matsushita M, Ushiama T. Two autopsy cases of Creutzfeldt-Jakob disease. Adv Neurol Sci (Japan) 1972; 16:452-9.

3 Rossum van A. Spastic pseudosclerosis. In: Vinken PJ and Bruyn GW ed. Vol. VI, Handbook of Clinical Neurology 1968; 726-60. Amsterdam: North-Holland Pub Co.

4 Mizutani T. Creutzfeldt-Jakob disease with cerebellar cortical degeneration-Special reference to a subtype of Creutzfeldt-Jakob disease with severe cerebrocerebellar atrophy. Adv Neurol Sci (Japan) 1977; 21:135-43, Excerpta med, Sect. VIII, 1977; 41:542.

5 Kirschbaum WR. Jakob-Creutzfeldt Disease. New York: American Elsevier, 1968.

6 Okumura A, Oda M, Iwase S, Shiraki H. Pathomorphological and clinicopathological study of grumose degeneration in the cerebellar dentate nucleus. Adv Neurol Sci (Japan) 1975; 19:483-92.

7 Shiraki H, Okumura A, Oyanagi S. Neuropathology of "grumose degeneration" of the cerebellar dentate nucleus with special reference to 
certain neurotoxic disorders and other pathological processes. In: Roizin L, Shiraki $H$ and Grčević M ed. Neurotoxicology, Vol. 1, 1977; 43-55, New York: Raven Press.

8 Shiraki H. Adult-pediatrics from neuropathological view point. From pediatric disorders to geriatric illnesses and vice versa. Jap J Pediat Med 1980; 12:349-58.

9 Creutzfeldt HG. Über eine eigenartige herdförmige Erkrankung des Zentralnervensystems. $Z$ ges Neurol Psychiat 1920; 57:1-19.

10 Jakob A. Über eigenartige Erkrankungen des Zentralnervensystems mit bemerkenswertem anatomischem Befunde. $Z$ ges Neurol Psychiat 1921; 64:147-228.

11 Gibbs CJ Jr, Gajdusek DC, Asher DM et al. Creutzfeldt-Jakob disease (Spongiform encephalopathy); Transmission to the chimpanzee. Science 1968; 161:388-9.

12 Jacob H, Eicke W, Orthner H. Zur Klinik und Neuropathologie der subakuten presenilen spongiösen Atrophien mit dyskinetischem Endstadium. Dtsch $Z$ Nervenheilk 1958; 178:330-57.

13 Nevin S, McMenemy WH, Behrman S, Jones DP. Subacute spongiform encephalopathy-A subacute form of encephalopathy attributable to vascular dysfunction (Spongiform cerebral atrophy). Brain 1960; 83:519-64.

14 Siedler H, Malamud N. Creutzfeldt-Jakob disease. Clinicopathologic report of 15 cases and review of the literature (with special reference to a related disorder designated as subacute spongiform encephalopathy). J Neuropath Exp Neurol 1963; 22:381-402.

15 Brownell B, Oppenheimer DR. An ataxic form of subacute presenile polioencephalopathy (Creutzfeldt-Jakob disease). J Neurol Neurosurg Psychiatry 1965; 28:350-361.

16 Beck E, Danniel PM, Matthews WB et al. Creutzfeldt-Jakob disease. The neuropathology of transmission experiment. Brain 1969; 92:699-716.

17 Masters CI, Richardson EP Jr. Subacute spongiform encephalopathy (Creutzfeldt-Jakob disease). The nature and progression of spongiform change. Brain 1978; 101:333-44.

18 Foley JM, Denny-Brown D. Subacute progressive encephalopathy with bulbar myoclonus. Excerpta med, Sect. VIII 1955; 782-4.

19 Silberman J, Vraviota H, Feigin I. Corticostriatal degeneration of the Creutzfeldt-Jakob type. J Neuropath Exp Neurol 1961; 20:105-18.

20 Katzman R, Kagan EH, Zimmerman HA. A case of Jakob-Creutzfeldt disease. J Neuropath Exp Neurol 1961; 20:78-94.
21 Tateishi J, Ohta M, Koga M, Sata Y, Kuroiwa Y. transmission of chronic spongiform encephalopathy with Kuru plaques from humans to small rodents. Ann Neurol 1979; 5:581-4.

22 Ohta M, Koga M, Tateishi J, Motomura S, et al. An autopsy case of spongiform encephalopathy associated with Kuru plaques and leukomalacia. Adv Neurol Sci (Japan) 1978; 22:487-96.

23 Nakashima K, Makino T, Kinoshita J, Yagishita S. A peculiar case of panencephalopathy with widespread distribution of plaques, status spongiosus and demyelination. Adv Neurol Sci (Japan) 1976; 20:362-71.

24 Hirano T, Tsuchiyama H, Kawai K, Mori K. An autopsy case of Creutzfeldt-Jakob disease with Kuru-like neuropathological changes. Acta Path Jap 1977; 27:231-8.

25 Nakamura T, Takamatsu I, Shida K, Kotorii K, Anraku S, Kida H. A case of subacute spongiform encephalopathy with numerous Kuruplaques in the cerebral and cerebellar cortices. Adv Neurol Sci (Japan) 1979; 23:484-92.

26 Chou SM, Martin JD. Kuru-plaques in a case of Creutzfeldt-Jakob disease. Acta Neuropath 1971; 17:150-5.

27 Horoupian DS, Powers JM, Schaumberg HH. Kuru-like neuropathological changes in a North American. Arch Neurol 1972; 27:555-61.

28 Krücke W, Beck E, Vitztum HG. CreutzfeldtJakob disease; Some unusual morphological features reminiscent of Kuru. J Neurol 1973; 206:1-24.

29 Adams H, Beck E, Shenkin AM. CreutzfeldtJakob disease. Further similarities with Kuru. J Neurol Neurosurg Psychiatry 1974; 35:195-200.

30 Zarranz JJ, Rivera Posman JM, Salisachs P. Kuru plaques in the brain of two cases with Creutzfeldt-Jakob disease. A common origin for the two diseases? J Neurol Sci 1979; 43:291-300.

31 May WW. Creutzfeldt-Jakob disease. 1 Survey of the literature and clinical diagnosis. Acta Neurol Scandinav 1968; 44:1-32.

32 Matsuoka T. Creutzfeldt-Jakob disease. Clinical manifestations and pathological features. $A d v$ Neurol Sci (Japan) 1974; 18:33-45.

33 Shiraki $H$. The neuropathological background for Creutzfeldt-Jakob disease (Creutzfeldt-Jakob syndrome). Adv Neurol Sci (Japan) 1974; 18:4-30.

34 Shiraki H. Slow virus infections of nervous system in humans mainly from neuropathological viewpoint. With special reference to comparison of so-called slow transmissible disease group to encephalomyelitides with conventional virus infections. Adv Neurol Sci (Japan) 1975; 19:109-47. 